\title{
Percepciones de autoevaluación: Autoestima, autoeficacia y satisfacción vital en la adolescencia
}

\author{
$\mathbf{M}^{\mathrm{a}}$ del Carmen Reina*, Alfredo Oliva, and Águeda Parra \\ Universidad de Sevilla \\ (Received March 23, 2010; Accepted April 27, 2010)
}

RESUMEN: En este estudio se analizan las relaciones entre tres variables relativas a la autoevaluación como son la autoestima, la autoeficacia y la satisfacción vital, así como sus tendencias evolutivas y las diferencias de género en una muestra de 2400 adolescentes con edades comprendidas entre los 12 y 17 años pertenecientes a 20 centros educativos de Andalucía Occidental. Por otro lado, se presentan las relaciones de estas tres variables con el estilo educativo parental. Los resultados indicaron correlaciones significativas entre las variables de autoevaluación, así como con las dimensiones del estilo parental. Concretamente, los niveles de afecto-comunicación y el sentido del humor de madres y padres fueron aspectos que se relacionaron de forma muy significativa con la autoevaluación adolescente. Por otra parte, aparecieron importantes diferencias de género en las trayectorias que la autoestima, la autoeficacia y la satisfacción vital siguen durante la adolescencia, ya que mientras que en los chicos se observaron pocos cambios, en las chicas la tendencia fue descendente, de forma que las adolescentes de más edad mostraron las autovaloraciones más bajas.

Palabras clave: autoestima, autoeficacia, satisfacción vital, estilo parental, diferencias de género.

Adolescents own perceptions of self-evaluation: Self esteem, self efficacy and life satisfaction ABSTRACT: In this study we analyse the relationships between three variables of self-evaluations, being self esteem, self efficacy and life satisfaction. Moreover, we study the evolution of these three aspects during adolescence paying attention to gender differences. The sample was made up of 2400 teenagers aged between 12 and 17 years old taken from 20 high schools in Western Andalusia. In this study we also analyse the relationship between teenager self evaluation and parenting style. Our results show, on one hand, high correlation between self esteem, self efficacy and life satisfaction, and on the other hand, between teenager self evaluation and parenting style. In fact, parental warmth and communication and sense of humor show high correlation with adolescent self evaluation. We also found increasing differences between boys and girls as regards their self esteem, self efficacy and life satisfaction as adolescence progressed. Specifically, little changed was observed in boys, whereas girls' self-evaluation decreased with age.

Key words: Self esteem, self efficacy, life satisfaction, parenting style, gender differences.

El reciente surgimiento de nuevos modelos centrados en la psicología o el desarrollo positivo ha supuesto un aumento del interés por algunos constructos 
psicológicos clásicos relacionados con el bienestar personal, tales como la autoestima, la autoeficacia y la satisfacción vital (Oliva et al., 2010). Aunque se trata de conceptos diferenciados, todos ellos se refieren a la percepción o evaluación que todo individuo hace de sí mismo, y tienen una carga afectiva evidente, por tratarse de conceptos relativos a la forma de percibir y valorar distintos aspectos personales. Ello hace que resulte interesante analizar las relaciones que estos tres aspectos autovalorativos tienen entre sí y con otras variables, especialmente en un periodo como la adolescencia, en el que chicos y chicas tendrán que hacer frente a diversos cambios y tareas evolutivas que repercutirán en la percepción que tienen de sí mismos (Havighurst, 1972).

La autoestima es considerada como un aspecto evaluativo del autoconcepto basado en la percepción global que el individuo tiene de su persona (Rosenberg, 1986), lo que no excluye la existencia de autoestimas parciales referidas a distintas facetas o dominios (Harter, 1998). La importancia de la autoestima viene avalada por el hecho de que puede considerarse como uno de los más potentes predictores del grado de ajuste psicológico durante la adolescencia y la adultez (Parra, Oliva, y Sánchez-Queija, 2004). Una autoestima positiva facilita a los jóvenes tener un buen ajuste psicológico, lo que a su vez favorece su adaptación social y puede mediar en la prevención de determinadas conductas de riesgo. Por tanto, podemos decir que la autoestima podría tener un efecto positivo en la satisfacción vital.

La autoeficacia tiene un alcance menos global puesto que hace referencia a la percepción de la propia capacidad para organizar y ejecutar las acciones necesarias para conseguir determinados logros o resultados (Bandura, 1997). Mientras que la autoestima es la autovaloración personal, la autoeficacia tiene que ver con la percepción que tiene un individuo acerca de su capacidad para conseguir un objetivo. No obstante, existen razones para pensar que ambos aspectos guardan relación entre sí, ya que el sentimiento de autoeficacia va a influir en el grado de implicación y persistencia del sujeto en la realización de tareas de cierta complejidad, lo que afectará en su resolución, con la consiguiente repercusión sobre su autoestima (Schunk y Meece, 2006). La autoeficacia también se relacionaría con la satisfacción vital ya que contribuye al logro de los objetivos personales con el consiguiente bienestar (Brannon, 2001). Al igual que la autoestima, la autoeficacia puede tanto vincularse a dominios específicos -por ejemplo, el ámbito académico- como valorarse de forma global.

Finalmente, la satisfacción vital refleja el bienestar subjetivo personal o el grado en que un individuo valora de forma favorable su calidad de vida (Veenhoven, 1995), por lo que ha sido considerado como el componente cognitivo del bienestar psicológico. Su importancia reside en la abundante evidencia empírica que indica que la satisfacción vital en la infancia y adolescencia se relaciona con indicadores de funcionamiento adaptativo (Huebner, 2004). En cierta medida, esta satisfacción con la propia vida es el resultado de una combinación entre la percepción que tiene el sujeto de sí mismo y de su entorno a nivel micro y macrosocial, por lo que algunas variables como la autoestima y algunos rasgos 
de la personalidad están en la base de la satisfacción vital (Diener, Suh, Lucas, y Smith, 1999).

En cuanto a la relación que estas autovaloraciones tienen con el género de los adolescentes, la mayor parte de las investigaciones encuentran diferencias significativas en la autoestima y observan menores puntuaciones en las chicas (Chabrol et al., 2004; Khanlou, 2004; Walker, 2000), aunque en algunos estudios la magnitud de las diferencias es baja (Matud, Ibáñez, Marrero, y Carballeira, 2003). Menos concluyentes son los resultados referentes a las diferencias de género en autoeficacia, ya que mientras que algunos estudios encuentran niveles más altos en los chicos (Anderman y Young, 1994; Meece y Jones, 1996), otros muestran cómo las chicas se sitúan por encima (Britner y Pajares, 2001). Tampoco faltan los que no hallan diferencias (Smith, Sinclair, y Chapman, 2002). Esta falta de consenso puede deberse tanto a las diferentes medidas utilizadas como a los tramos de edad analizados, ya que las diferencias entre chicos y chicas pueden cambiar a lo largo de la adolescencia. Por último, las diferencias en satisfacción vital parecen favorecer ligeramente a los varones (Goldbeck, Schmitz, Besier, Herschbach, y Herich, 2007), aunque tampoco faltan los estudios que no hallan diferencias (Ash y Huebner, 2001; Huebner, Drane, y Valois 2000).

En lo referente a la tendencia evolutiva a lo largo de la adolescencia, el consenso tampoco es total, apareciendo, además, discrepancias en función del tipo de autovaloración considerada. Si nos referimos a la autoestima, algunas investigaciones coinciden en encontrar un descenso durante la adolescencia temprana, que tiende a recuperarse en los años posteriores, especialmente en el caso de los chicos, ya que en las chicas los niveles de autoestima suelen mantenerse bajos durante toda la adolescencia (Parra et al., 2004; Savin-Williams y Demo, 1984). También se observa una tendencia similar en la autoeficacia, con un descenso significativo en la adolescencia temprana, sobre todo en estudios que evalúan autoeficacia relativa a materias escolares, por lo que el descenso puede atribuirse a los cambios en el contexto escolar que tienen lugar con el paso de la escuela primaria a la secundaria o instituto (Bandura, 2006; Eccles, Wigfield, y Schiefele, 1998; Urdan y Midgley, 2003). Algo diferente es la tendencia mostrada por la satisfacción vital, ya que parece ser una variable muy estable, a tenor de lo que indican los estudios realizados con población adolescente (Huebner, 1997; Huebner et al., 2000).

En cuanto a los factores asociados a la autovaloración durante la adolescencia, son numerosos los estudios que señalan la relación entre el estilo educativo parental y la autoestima o la satisfacción vital, mostrando que los chicos y chicas que se sienten apoyados y queridos por sus progenitores son quienes obtienen puntuaciones más altas en escalas que miden estos constructos, aunque otras dimensiones parentales, como el uso de técnicas de control inductivas o la promoción de autonomía, también se han asociado positivamente a las autovaloraciones favorables (Ash y Huebner, 2001; Lee, Daniels, y Kissinger, 2006). Aunque la evidencia es menor, algunos estudios han encontrado relación entre algunas 
prácticas familiares estimulantes y democráticas y el sentimiento de autoeficacia (Bandura, 2006; Schunk y Meece, 2006).

Teniendo en cuenta todo lo anterior, el objetivo principal de este estudio fue analizar las relaciones entre las variables de autoestima, autoeficacia y satisfacción vital, así como las asociaciones que establecen dichas variables con la edad, el género y con las dimensiones del estilo parental. La originalidad de este estudio radica en la consideración conjunta de tres variables referidas a la autovaloración y en haber analizado la relación de estas tres variables con varias dimensiones del estilo parental.

Las hipótesis de las que partimos son varias: en primer lugar, la existencia de correlaciones positivas entre las variables de autopercepción contempladas en el estudio. En segundo lugar, esperamos encontrar diferencias de género a favor de los chicos, en el sentido de presentar autopercepciones más positivas que las chicas. Finalmente, creemos que existen relaciones entre las variables de estilo parental y las de autopercepción. Así, creemos que las percepciones más favorables serán las de los chicos y chicas que perciben estilos parentales caracterizados por el afecto, la comunicación, el humor, la promoción de la autonomía y la falta de control psicológico. Con respecto a la influencia de la edad, no teníamos una hipótesis de partida clara.

\section{MÉTODO}

\section{Participantes}

Se seleccionaron 20 centros educativos de Andalucía Occidental. Con el objetivo de captar la mayor diversidad posible realizamos un muestreo intencional fijando una serie de cuotas: tamaño del municipio y zona (rural o urbana) en la que se localizaban los centros escolares, tamaño de los centros, titularidad pública o privada, y nivel socioeconómico de la barriada en la que estaba enclavado el colegio o instituto.

La muestra estuvo constituida por 2400 adolescentes que cursaban estudios de $2^{\circ}, 3^{\circ}$ y $4^{\circ}$ de ESO, Bachillerato y Ciclos Formativos de grado medio. Fueron 1068 chicos $(44.5 \%)$ y 1332 chicas $(55.5 \%)$ con edades comprendidas entre 12 y 17 años $(M=14,73, D T=1,25)$. Un $25.5 \%$ de adolescentes cursaba $2^{\circ}$ de ESO; un $26.8 \%, 3^{\circ}$ de ESO y un $28.7 \%$ cursaba $4^{\circ}$ de ESO. En niveles educativos superiores, Bachillerato y Ciclos Formativos de Grado Medio, al igual que sucede en la población de procedencia, la proporción de alumnado fue menor en comparación con el nivel obligatorio, cursando un $18.2 \% 1^{\circ}$ de $\mathrm{Ba}-$ chillerato, mientras que el $0.8 \%$ del alumnado realizaba el $1^{\text {er }}$ curso de un Ciclo Formativo.

En relación a características sociodemográficas, es relevante mencionar que la nacionalidad mayoritaria del alumnado era española (97.8\%), al igual que la de sus progenitores $(96.6 \%)$. Atendiendo a la estructura familiar, la mayoría del alumnado (un $84.8 \%$ ) procedía de familias formadas por dos progenitores. 


\section{Instrumentos}

Escala de Autoestima. Para la valoración de la autoestima global se utilizó la Escala de Autoestima de Rosenberg (1965), compuesta por 10 ítems de tipo Likert (e.g., "a veces pienso que no sirvo absolutamente para nada"), con opciones de respuesta comprendidas entre 1 (totalmente en desacuerdo) y 4 (totalmente de acuerdo). El coeficiente de fiabilidad, alfa de Cronbach, para la escala fue de .82. La adaptación española de esta escala ha sido validada tanto en población adolescente (Atienza, Moreno, y Balaguer, 2000) como universitaria (MartínAlbo, Nuñez, Navarro, y Grijalvo, 2007).

Escala de Autoeficacia Generalizada. La estimación de la autoeficacia se ha realizado con la adaptación española de la Escala de Autoeficacia General (Baessler y Schwarzer 1996), validada en nuestro país por Sanjuán, Pérez, y Bermúdez (2000). Este instrumento es una escala unidimensional compuesta por 10 ítems (e.g., "puedo encontrar la manera de obtener lo que quiero aunque alguien se me oponga"), que deben ser puntuados en una escala tipo Likert comprendida entre 1 (incorrecto) y 4 (cierto). El coeficiente de fiabilidad, alfa de Cronbach, para la escala fue de .82 .

Escala para la Evaluación de la Satisfacción Vital. La Student's Life Satisfaction Scale fue elaborada por Huebner (1991). Es un instrumento que sirve para evaluar la satisfacción vital de los chicos y chicas adolescentes, y cuya versión española ha sido validada recientemente (Galíndez y Casas, 2010). Está compuesta por siete ítems (e.g., "tengo lo que quiero en la vida") que deben ser puntuados en una escala tipo Likert comprendida entre 1 (totalmente en desacuerdo) y 7 (totalmente de acuerdo). No dispone de subescalas, por lo que contempla tan sólo una puntuación global. El coeficiente de fiabilidad, alfa de Cronbach, para esta escala fue de .81

Escala para la evaluación del Estilo Parental. Esta escala fue elaborada por Oliva, Parra, Sánchez-Queija, y López (2007), para evaluar la percepción que chicos y chicas adolescentes tienen de diversas dimensiones del estilo educativo de sus padres y madres. Está compuesta por 41 ítems que deben ser puntuados en una escala tipo Likert comprendida entre 1 (totalmente en desacuerdo) y 6 (totalmente de acuerdo). En esta escala se agrupan las siguientes dimensiones: Afecto y comunicación: Se refiere a la expresión de apoyo y afecto por parte de los padres, a su disponibilidad y a la fluidez de la comunicación con ellos. Está compuesta por ocho ítems, (e.g., "si tengo algún problema puedo contar con su ayuda"); el coeficiente de fiabilidad, alfa de Cronbach, para la subescala fue de 92. Promoción de autonomía: Esta dimensión evalúa en qué medida padre y madre animan a su hijo para que tenga sus propias ideas y tome sus propias decisiones. Se compone de ocho ítems, (e.g., "me anima a que diga lo que pienso aunque él/ella no esté de acuerdo"); alfa de Cronbach $=.88$. Control conductual: Esta dimensión incluye seis ítems que se refieren al establecimiento de límites y a los intentos de los padres por mantenerse informados sobre el comportamiento de sus hijos e hijas fuera de casa (e.g., "pone límites a la hora a la que debo volver 
a casa"); alfa de Cronbach $=.82$. Control psicológico: Evalúa la utilización por parte de padres y madres de estrategias manipuladoras como el chantaje emocional y la inducción de culpa, por lo que es una dimensión claramente negativa. Tiene ocho ítems (e.g., "me hace sentir culpable cuando no hago lo que quiere") y una fiabilidad de alfa de Cronbach de .86. Revelación: Incluye ocho ítems que indagan sobre la frecuencia con que los adolescentes cuentan a sus padres asuntos personales por propia iniciativa (e.g., "le cuento lo que hago en mi tiempo libre"); obteniendo un índice de fiabilidad, alfa de Cronbach de .85. Humor: Esta dimensión indica en qué medida el adolescente considera que sus padres muestran optimismo y buen sentido de humor. Se compone de seis ítems (e.g., "casi siempre es una persona alegre y optimista") y alcanza una fiabilidad de alfa de Cronbach de .88 .

\section{Procedimiento}

Los objetivos del estudio fueron explicados al director/a de los centros elegidos en contactos previos, después de los cuales dos miembros del equipo de investigación los visitaron y aplicaron los instrumentos en las aulas elegidas, que fueron todas las correspondientes a los niveles de ESO (salvo 1er curso), Bachillerato y Ciclos Formativos descritos líneas atrás. Los padres fueron informados por correo del estudio, y ningún padre se opuso al mismo. Para garantizar la sinceridad y privacidad de las respuestas los alumnos de cada una de las clases seleccionadas cumplimentaron el cuestionario de forma voluntaria y anónima. Lo hicieron en una sesión de una hora de duración en presencia de un miembro del equipo investigador.

\section{RESULTADOS}

Para responder a nuestros objetivos e hipótesis de investigación vamos, en primer lugar, a realizar análisis de correlaciones de las variables relativas a la autopercepción adolescente entre ellas mismas y con las referidas del estilo parental. A continuación, realizaremos análisis de regresión para conocer la contribución específica de las variables destacadas previamente en los análisis de correlaciones. Finalmente, para analizar las diferencias en autoestima, satisfacción vital y autoeficacia en función del sexo y la edad, llevaremos a cabo dos análisis de la varianza.

\section{Análisis de correlaciones}

A continuación presentamos las correlaciones de las variables de autoestima, autoeficacia y satisfacción vital entre ellas mismas y con las referidas al estilo parental (afecto y comunicación, promoción de autonomía, control conductual, control psicológico, revelación y sentido del humor).

Como puede observarse en la Tabla 1, las variables de autoestima, autoefi- 
cacia y satisfacción vital de los adolescentes mostraron muchas correlaciones significativas, entre ellas y con las relacionadas con el estilo parental. Podemos apreciar altas correlaciones positivas entre las variables de nuestro estudio, destacando las de la autoestima con la autoeficacia y con la satisfacción vital. Las variables relativas a la autoevaluación correlacionaron positivamente con las variables del estilo parental, siendo la única excepción el control psicológico, que correlacionó de forma negativa con las variables de autoestima, autoeficacia y satisfacción vital. La dimensión de afecto-comunicación resultó la variable que mostró las asociaciones positivas más fuertes con autoestima, autoeficacia y satisfacción vital, seguida, en el caso de la autoestima y la satisfacción vital, por la dimensión del humor, y en el caso de la autoeficacia por la promoción de autonomía. El control conductual fue la variable que menos relación mostró con la autoevaluación adolescente, ya que sólo correlacionó significativamente con la satisfacción vital.

Tabla 1. Correlaciones entre Medidas de las Variables de Autoevaluación y los Estilos Parentales

\begin{tabular}{|c|c|c|c|c|c|c|c|c|}
\hline & 1 & 2 & 3 & 4 & 5 & 6 & 7 & 8 \\
\hline \multicolumn{9}{|l|}{ 1. Autoestima } \\
\hline 2. Autoeficacia & $.49 * * *$ & & & & & & & \\
\hline 3. Satisfacción vital & $.49 * * *$ & $.33 * * *$ & & & & & & \\
\hline 4. Afecto comunicación & $.24 * * *$ & $.17 * * *$ & $.36 * * *$ & & & & & \\
\hline 5. Promoción de autonomía & $.19 * * *$ & $.17 * * *$ & $.28 * * *$ & $.63 * * *$ & & & & \\
\hline 6. Control conductual & .02 & .03 & $.06^{* *}$ & $.23 * * *$ & $.22 * * *$ & & & \\
\hline 7. Control psicológico & $-.19 * * *$ & $-.04 *$ & $-.27 * * *$ & $-.35 * * *$ & $-.32 * * *$ & $.22 * * *$ & & \\
\hline 8. Revelación & $.12 * * *$ & $.10 * * *$ & $.23 * * *$ & $.49 * * *$ & $.47 * * *$ & $.33 * * *$ & $-.16^{* * *}$ & \\
\hline 9. Humor & $.22 * * *$ & $.16^{* * *}$ & $.35 * * *$ & $.66^{* * *}$ & $.59 * * *$ & $.15 * * *$ & $-.36 * * *$ & $.52 * * *$ \\
\hline
\end{tabular}

$* * * \mathrm{p}<.001,{ }^{* *} \mathrm{p}<.01,{ }^{*} \mathrm{p}<.05$

\section{Análisis de regresión}

Para analizar más a fondo las relaciones de la autoestima, la autoeficacia y la satisfacción vital adolescente con los estilos parentales se realizó un análisis de regresión sobre cada una de las variables analizadas.

Como puede observarse en la Tabla 2, el sexo se asoció de manera significativa con las tres variables estudiadas, ya que los chicos puntuaron más alto que las chicas en dichas variables. La edad no influyó significativamente en ninguna de las variables estudiadas. El afecto/comunicación fue la variable que más contribuyó a explicar las diferencias en la percepción de autovaloración, pues se asoció positivamente con las tres variables criterio. La percepción de mejor humor en los estilos educativos de los padres se relacionó de forma significativa con la autoestima y la satisfacción vital. Por el contrario, el control psicológico se asoció negativamente con la autoestima y la satisfacción vital. La promoción de autonomía contribuyó de forma significativa a la autoeficacia, mientras que la 
revelación se relacionó de forma positiva con la satisfacción vital. Finalmente, el control conductual no realizó ninguna aportación significativa a la autoevaluación adolescente.

Tabla 2. Regresión Múltiple sobre las Variables de Autoevaluación

\begin{tabular}{|c|c|c|c|c|c|c|}
\hline \multirow[b]{2}{*}{ Predictores } & \multicolumn{2}{|c|}{ Autoestima } & \multicolumn{2}{|c|}{ Autoeficacia } & \multicolumn{2}{|c|}{ Satisfacción vital } \\
\hline & Beta & $\begin{array}{l}\mathrm{R}^{2} \mathrm{y} \text { sign. } \\
\text { del cambio }\end{array}$ & Beta & $\begin{array}{l}\mathrm{R}^{2} \mathrm{y} \text { sign. } \\
\text { del cambio }\end{array}$ & Beta & $\begin{array}{l}\mathrm{R}^{2} \text { y sign. } \\
\text { del cambio }\end{array}$ \\
\hline & & $.12 * * *$ & & $.08 * * *$ & & $.18^{* * *}$ \\
\hline Sexo & $-.21 * * *$ & & $-.20 * * *$ & & $-.09 * * *$ & \\
\hline Edad & -.03 & & -.01 & & -.03 & \\
\hline Afecto y comunicación & $.12^{* * *}$ & & $.08 * *$ & & $.17 * * *$ & \\
\hline Promoción de autonomía & .04 & & $.11 * * *$ & & .01 & \\
\hline Control conductual & .02 & & -.01 & & .02 & \\
\hline Control psicológico & $-.13^{* * *}$ & & .04 & & $-.16^{* * *}$ & \\
\hline Revelación & .00 & & .01 & & $.05^{*}$ & \\
\hline Humor & $.07 * *$ & & .06 & & $.14^{* * *}$ & \\
\hline
\end{tabular}

${ }^{* * *} \mathrm{p}<.001,{ }^{* *} \mathrm{p}<.01,{ }^{*} \mathrm{p}<.05$

\section{Análisis de la varianza}

Seguidamente presentamos las relaciones de las variables de autoestima, autoeficacia y satisfacción vital con el sexo adolescente. En la Tabla 3 se presentan las puntuaciones medias de estas variables y los resultados de los ANOVAS que comparan los dos grupos. Las diferencias fueron claramente significativas. Siendo las medias de los chicos más altas en las tres variables estudiadas.

Tabla 3. Diferencias en la Autoevaluación Adolescente en Función del Sexo

\begin{tabular}{lcccc}
\hline & Hombre & Mujer & $F$ & $\mathrm{Eta}^{2}$ \\
\hline 1. Autoestima & 3.26 & 3.07 & $90.94^{* * *}$ & .04 \\
2. Autoeficacia & 3.10 & 2.92 & $83.21^{* * *}$ & .03 \\
3. Satisfacción vital & 5.30 & 5.16 & $9.45^{* *}$ & .00
\end{tabular}

${ }^{* * *} \mathrm{p}<.001,{ }^{* *} \mathrm{p}<.01,{ }^{*} \mathrm{p}<.05$

En las Tabla 4 y 5 se presentan las puntuaciones medias de las variables de autoevaluación en relación con la edad y los resultados de los ANOVAs que comparan los tres grupos, para chicos y chicas por separado. Puede observarse como en el caso de los varones las diferencias no fueron significativas para ninguna de las tres variables. Sin embargo, cuando se trataba de las chicas, se 
observó un descenso significativo en las puntuaciones en autoestima y satisfacción vital a lo largo de la adolescencia. Los análisis post hoc indicaron que había diferencias significativas en ambas variables, entre el grupo de edad de 12-13 años y el de 16-17 años.

Tabla 4. Autoevaluación de los Chicos en Función de la Edad

\begin{tabular}{lccccc}
\hline & $\begin{array}{c}\text { Edad } \\
12-13\end{array}$ & $\begin{array}{c}14-15 \\
N=223\end{array}$ & $\begin{array}{c}16-17 \\
N=518\end{array}$ & $F=327$ & $\mathrm{Eta}^{2}$ \\
& 3.26 & 3.27 & 3.24 & 0.31 & .00 \\
1. Autoestima & 3.09 & 3.11 & 3.10 & 0.07 & .00 \\
2. Autoeficacia & 5.32 & 5.31 & 5.28 & 0.12 & .00 \\
3. Satisfacción vital & & & & &
\end{tabular}

Tabla 5. Autoevaluación de las Chicas en Función de la Edad

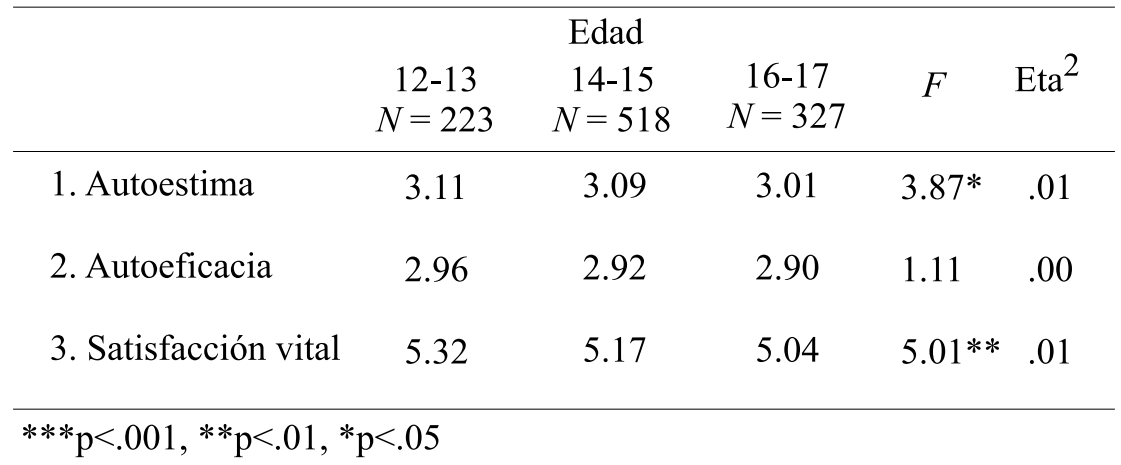

\section{DISCUSIÓN}

Como se había previsto, las puntuaciones en autoestima, autoeficacia y satisfacción vital mostraron correlaciones muy significativas, aunque en ningún caso fueron superiores a .50. Ello pone de manifiesto que aunque se trata de aspectos relativos a la autoevaluación del adolescente, son facetas diferenciadas que tiene sentido mantener independientes. Correlaciones similares han sido encontradas en estudios llevados a cabo sobre población adulta (De Neve, y Cooper, 1998; Houston, McKee, y Wilson, 2000; MacDonald, Piquero, Valois, y Zullig, 2005). Aunque si bien es cierto que la correlación entre la autoeficacia y la satisfacción 
vital resultó significativa, fue claramente de menor magnitud que las correlaciones que la autoestima estableció con las otras dos variables lo que bien pudiera deberse a que mientras que la autoestima es una autovaloración global, la autoeficacia se refiere a la valoración que hace el sujeto de un aspecto mucho más concreto, como es su capacidad para alcanzar objetivos, por lo que es lógico que tenga una menor influencia sobre la satisfacción vital.

En cuanto a la tendencia que a partir de la pubertad siguen las tres variables de autoevaluación, se han encontrado diferencias de género favorables a los varones en autoestima y satisfacción vital, ya que mientras que entre ellos los valores en estas variables se mantuvieron estables, en el caso de las chicas se produjo un decremento significativo durante la adolescencia, de forma que las diferencias de género fueron más acusadas en la última etapa (Chabrol et al., 2004; Parra et al., 2004; Walker, 2000). Aunque los valores en autoeficacia mostraron mucha estabilidad absoluta en el tramo de edad estudiado, también en este caso los varones obtuvieron puntuaciones más elevadas. Esta mayor autoestima y satisfacción vital en los varones, que coincide con lo encontrado en otros estudios (Goldbeck et al., 2007; Khanlou, 2004), podría atribuirse al mayor impacto negativo que muchos de los cambios físicos propios de la pubertad tienen sobre las chicas (Susman y Dorn, 2009), sin olvidar que aún se mantienen diferencias de género en los procesos de socialización a nivel familiar y cultural, que hacen que a partir de la pubertad el abanico de posibilidades y experiencias (llegar más tarde a casa o ir de vacaciones con los amigos) se amplíe de forma más clara para los chicos que para las chicas, que incluso llegan a experimentar algunas restricciones como consecuencia de su nuevo estatus puberal (Oliva, 2006). Menos consenso hay con respecto a las diferencias de género en autoeficacia, aunque cuando se trata de una eficacia no exclusivamente referida al ámbito académico los chicos suelen situarse por encima, como ha sido nuestro caso (Anderman y Young, 1994; Meece y Jones, 1996). Estas diferencias de género en las medidas de autoevaluación resultan muy preocupantes si tenemos en cuenta la evidencia empírica que indica que una pobre autoestima o satisfacción vital suele asociarse a problemas de ajuste psicológico y social, ya que sitúa a las chicas en una posición de mayor vulnerabilidad en la adolescencia media y tardía. En este sentido, y como indican los estudios epidemiológicos, la prevalencia de trastornos psicológicos suele aumentar durante la adolescencia, sobre todo entre las chicas (Graber, 2004).

Otro resultado de este estudio que merece la pena resaltar es la contribución que las variables o dimensiones del estilo parental realizaron a la hora de explicar la variabilidad en las puntuaciones de las variables de autoevaluación. Cada dimensión mostró distinto peso en función de la variable de autoevaluación concreta, aunque fueron el afecto/comunicación y el humor las variables más determinantes. Así, cuando el afecto y la comunicación caracterizan las relaciones entre padres y adolescentes, éstos últimos muestran una mejor autoestima y satisfacción vital, así como una mejor autoeficacia, lo que no hace sino apoyar la evidencia empírica disponible (Noller y Callan, 1991). A su vez, un estilo pa- 
rental caracterizado por una actitud alegre, relajada y optimista, es decir el buen humor, contribuye a generar un clima familiar que favorece la socialización promoviendo el bienestar de niños y adolescentes (Darling y Steinberg, 1993; Dunn, 1993; Oliva, Parra, y Arranz, 2008).

Así mismo, los padres que favorecen la autonomía contribuyen al desarrollo de la autoeficacia en sus hijos, ya que suelen usar prácticas educativas que promueven una mayor capacidad para pensar, formar opiniones propias y tomar decisiones, consiguiendo fomentar un mayor sentimiento de autoeficacia (Allen, Hauser, Eickholt, Bell, y O’Connor, 1994).

En cambio, el control psicológico, basado en la utilización de tácticas disciplinarias que se sirven del chantaje emocional o la inducción de culpa, se relacionó negativamente con las variables de autoestima y satisfacción vital. Esto coincide con los resultados de otras investigaciones en las que el control psicológico apareció relacionado a un pobre ajuste emocional (Silk, Morris, Kanaya, y Steinberg, 2003). En cuanto al control conductual, que incluía el establecimiento de límites y la monitorización de la conducta del adolescente, no mostró relaciones significativas con ninguna de las tres variables de autovaloración, probablemente porque el control es más eficaz para la prevención de los problemas comportamentales que para promover la autoestima o el bienestar psicológico (Oliva, 2006).

Los resultados de este estudio permiten extraer algunas conclusiones de cara a la intervención. Así, el hecho de que las chicas obtengan puntuaciones más bajas en autoestima y satisfacción vital sugiere la necesidad de prestar una atención especial a estas diferencias de género y nos alerta de la necesidad de trabajar estos aspectos con ellas. La autoestima es uno de los indicadores más potentes de ajuste psicológico y adaptación social, por lo que es fundamental aumentar el bienestar que las jóvenes sienten con ellas mismas por ejemplo a través de programas de aprendizaje socio-emocional. Así, estaremos contribuyendo a mejorar su salud para el futuro y su calidad de vida. Por otro lado, sería importante promover entre madres y padres de adolescentes estilos parentales caracterizados por el afecto, la comunicación y la ausencia de control psicológico, ya que estas variables se mostraron muy relacionadas con autovaloraciones positivas de los adolescentes.

Entre las limitaciones de este estudio, hay que hacer referencia a su carácter transversal, que imposibilita el establecimiento de relaciones de causalidad entre el estilo parental y las medidas de autovaloración adolescente. Además, la utilización del adolescente como única fuente de información ha podido aumentar las correlaciones entre las variables estudiadas. En futuros estudios longitudinales sería interesante poder analizar la estabilidad temporal de las relaciones establecidas en el presente trabajo, así como utilizar otras fuentes de información tales como padres o profesores. No obstante, consideramos que los resultados de este estudio contribuyen a un mejor conocimiento de la relación entre las tres variables de autoevaluación y a su asociación con el estilo parental, lo que apoya el papel que la familia sigue desempeñando para el buen ajuste durante la adoles- 
cencia. Por otro lado, nuestros resultados vuelven a recordarnos las importantes diferencias de género que existen entre los adolescentes de nuestro contexto. Diferencias que se magnifican a medida que transcurren los años, situando a las mujeres en una posición de clara desventaja a las puertas de la adultez.

\section{AGRADECIMIENTOS}

Este estudio ha sido realizado gracias a la financiación de la Consejería de Salud de la Junta de Andalucía.

\section{REFERENCIAS}

Allen, J. P., Hauser, S., Eickholt, C., Bell, K., y O’Connor, T. (1994). Autonomy and relatedness in family interactions as predictors of expressions of negative adolescent affect. Journal of Research on Adolescence, 4, 535-552.

Anderman E. M., y Young, A. J. (1994). Motivation and strategy use in science: Individual differences and classroom effects. Journal of Research in Science Teaching, 3, 811-831.

Ash, C., y Huebner, E. S. (2001). Environmental events and life satisfaction reports of adolescents: A Test of Cognitive Mediation. School Psychology International, 22, 320-336.

Atienza, F. L., Moreno, Y., y Balaguer, I. (2000). Análisis de la dimensionalidad de la Escala de Autoestima de Rosenberg en una muestra de adolescentes valencianos. Revista de Psicología Universitas Tarraconensis, 22, 29-42.

Baessler, J., y Schwarzer, R. (1996). Evaluación de la autoeficacia: adaptación española de la escala de autoeficacia general. Ansiedad y Estrés, 2, 1-8.

Bandura, A. (1997). Self-Efficacy. The exercise of control. New Jersey: Freeman.

Bandura, A. (2006). Self-efficacy beliefs of adolescents. En F. Pajares, y T. Urdan (Eds.), Adolescence and Education (pp. 307-335). Greenwich, CT: Information Age Publishing.

Batista-Foguet, J., Fortiana, J., Currie, C., y Villalbi, J. (2004). Socio-economic indexes in surveys for comparisons between countries. Social Indicators Research, 37(3), 315-332.

Brannon, L. (2001). Psicología de la salud. Madrid: Thomson Paraninfo.

Britner, S. L., y Pajares, F. (2001). Self-efficacy beliefs, motivation, race and gender in middle school science. Journal of Women and Minorities in Sciences and Engineering, 7, 271-285.

Chabrol, H., Carlin, E., Michaud, C., Rey, A., Cassan, D., Juilot, M. et al. (2004). A study of the Rosenberg self-estem scale in a sample of high-school students. Neuropsychiatrie del'Enfance et del'Adolescence, 52, 533-536.

Darling, N., y Steinberg, L. (1993). Parenting style as context: an integrative model. Psychological Bulletin, 113, 487-496.

De Neve, K. M., y Cooper, H. (1998). The happy personality: A meta-analysis of 137 personality traits and subjective well-being. Psychological Bulletin, 


$$
\text { 124, 197-229. }
$$

Diener, E., Suh, E., Lucas, R., y Smith, H. (1999). Subjetive well-being: three decades of progress. Psychological Bulletin, 125, 276-302.

Dunn, J. (1993). Young children's close relationships: Beyond attachment. Newbury Park, CA: Sage.

Eccles, J. S., Wigfield, A., y Schiefele, U. (1998). Motivation to succeed. En W. Damon, y N. Eisenberg (Eds.), Handbook of child psychology (pp. 10171095). New York: Wiley.

Galíndez, E., y Casas, F. (2010). Adaptación y validación de la Students’ Life Satisfaction Scale (SLSS) con adolescentes. Estudios de Psicología, 31, 79-88.

Goldbeck, L., Schmitz, T. G., Besier, T., Herschbach, P., y Henrich, G. (2007). Life satisfaction during adolescence. Quality of Life Research, 16, 969-979.

Graber, J. A. (2004). Internalizing problems during adolescence. En R. M. Lerner, y Steinberg, L. (Eds.), Handbook of adolescent psychology (pp. 155-188). New York: Wiley.

Harter, S. (1998). The developmental of self-representation. En W. Damon, y N. Eisenberg (Eds.), Handbook of child psychology: Vol 3, Social, emotional and personality development (pp. 345-376). New York: Wiley.

Havighurst, R. J. (1972). Developmental tasks and education ( $3^{\mathrm{a}}$ ed.). New York: Longman.

Houston, D. M., McKee, K. J., y Wilson, J. (2000).Attributional style, efficacy, and the enhancement of well-being among housebound older people. Basic and Applied Social Psychology, 22, 309-317.

Huebner, E. S. (1991). Further validation of the Students Life Satisfaction Scale: The independence of satisfaction and affect rating. Journal of Psychoeducational Assessment, 9, 363-368.

Huebner, E. S. (1997). Life satisfaction and happiness. En G. Bear, K. Minke, y A. Thomas (Eds.), Children's needs II (pp. 271-278). Washington, DC: National Association of School Psychologists.

Huebner, E. S. (2004). Research on assessment of life satisfaction of children and adolescents. Social Indicators Research, 66, 3-33.

Huebner, E. S., Drane J. W., y Valois R. F. (2000). Levels and demographic correlates of adolescent life satisfaction reports. School Psychology International, 21, 281-292.

Martín-Albo, J., Núñez, J. L., Navarro, J. G., y Grijalvo, F. (2007). The Rosenberg Self-Esteem Scale: Translation and validation in University students. Spanish Journal of Psychology, 10, 458-467.

Meece, J. L., y Jones, M. G. (1996). Gender differences in motivation and strategy use in science: Are girls rote learners? Journal of Research in Science and Teaching, 33, 407-431.

Khanlou, N. (2004). Influences on adolescent self-esteem in multicultural Canadian schools. Public Health Nursing, 21, 404-411.

Lee, S. M., Daniels, M. H., y Kissinger, D. B. (2006). Parental influences on adolescent adjustment: parenting styles versus parenting practices. Family 
Journal: Counseling and Therapy for Couples and Families, 14, 253-259.

MacDonald, J., Piquero, A., Valois, R., y Zullig, K. (2005). The relationship between life satisfaction, risk-taking behaviors, and youth violence. Journal of Interpersonal Violence, 20, 1495-1518.

Maddux, J. E. (2005). Self-efficacy. En C. R. Snyder, y S. J. López (eds.), Handbook of Positive Psychology (pp. 277-287). Oxford: University Press.

Matud M. P., Ibáñez I., Marrero R. J., y Carballeira M. (2003). Diferencias en autoestima en función del género. Análisis y Modificación de Conducta, 29, 51-78.

Matud, M. P., Marrero, R. J., Carballeira, M., Pérez, M., Correa, M. L., Aguilera, B., y Sánchez, T. (2003). Transmisión intergeneracional de la violencia doméstica. Psicología Conductual, 11, 25-40.

Noller, P., y Callan, V. (1991). The adolescent in the family. Londres: Routledge.

Oliva, A. (2006). Relaciones familiares y desarrollo adolescente. Anuario de Psicología, 37, 209-223.

Oliva, A., Parra, A., y Arranz, A. (2008). Estilos relacionales materno y paterno y ajuste adolescente. Infancia y aprendizaje, 31, 93-106.

Oliva, A., Parra, A., Sánchez-Queija, I., y López. F. (2007). Estilos educativos materno y paterno: evaluación y relación con el ajuste adolescente. Anales de Psicología, 23, 49-56.

Oliva, A., Ríos, M., Antolín, L., Parra, A., Hernando, A., y Pertegal, A. (2010). Más allá del déficit: Construyendo un modelo de desarrollo positivo adolescente. Infancia y Aprendizaje, 33, 223-234.

Parra, A., Oliva, A., y Sánchez-Queija, I. (2004). Evolución y determinantes de la autoestima durante los años adolescentes. Anuario de Psicología, 35, 331346.

Rosenberg, M. (1965). Society and the adolescent self-image. Princeton, NJ: Princeton University Press.

Rosenberg, M. (1986). Self-concept from middle childhood through adolescence. En J. Suls, y A.G. Greenwald (Eds), Psychological perspectives on the self (Vol 3, pp. 107-136). Hillsdale NJ: Lawrence Erlbaum Associates, Inc.

Sanjuán, P., Pérez, A. M., y Bermúdez. J. (2000). Escala de autoeficacia general: datos psicométricos de la adaptación para población española. Psicothema, 12, 509-513.

Savin-Williams, R. C., y Demo, D. H. (1984). Development change and stability in adolescent self-concept. Development Psychology, 20, 1100-1110.

Schunk, D. H., y Meece, J. L. (2006). Self-efficacy in adolescence. En F. Pajares, y T. Urdan (Eds.), Adolescence and Education (Vol. 5, pp. 71-96). Greenwich, CT: Information Age Publishing.

Silk, J., Morris, A., Kanaya, T., y Steinberg, L. (2003). Psychological control and autonomy granting: Opposite ends of a continuum or distinct constructs? Journal of Research on Adolescence, 13, 113-128.

Smith, L., Sinclair, K. E., y Chapman, E. S. (2002). Students' goals, self-efficacy, self-handicapping, and negative affective responses: An Australian senior 
school student study. Contemporary Educational Psychology, 27, 471-485.

Susman, E. J., y Dorn, L. D. (2009). Puberty: Its role in development. En R. Lerner, y L. Steinberg (Eds.), Handbook of adolescent psychology $\left(3^{a} E d-\right.$ ición). Volume I: Individual bases of adolescent development (pp. 116-151). Hoboken, NJ: Wiley.

Urdan, T. C., y Midgley C. (2003). Changes in the perceived classroom goal structure and pattern of adaptive learning during early adolescence. Contemporary Educational Psychology, 28, 524 - 551.

Veenhoven, R. (1995). Developments in satisfaction research. Social Indicators Research, 37, 1-46.

Walker, H. R. (2000). An analysis of the relationship of human sexuality knowledge, self-esteem, and body image to sexual satisfaction in college and university students. Dissertation Abstract International: Section B: The Sciences and Engineering, 60, 45-60. 